Z. klin. Chem. u. klin. Biochem.

8. Jg., S. $492-497$, September 1970

\title{
Photometrische Bestimmung der Myeloperoxydase mittels $p$-Phenylendiamin
}

\author{
Von W. Vormittag und H. Pietschmann \\ Aus der II. Medizinischen Universitätsklinik in Wien (Vorstand: Prof. Dr. K. Fellinger)
}

(Eingegangen am 26. Januar 1970)

In einem photometrisch-kinetischen Test unter Verwendung von $p$-Phenylendiamin als Wasserstoffdonator wurde eine Reihe kinetischer Parameter sowohl für die Myeloperoxydase als auch für die Meerrettichperoxydase untersucht.

Unter bestimmten Versuchsbedingungen erivies sich die einfach und rasch durchführbare Methode zur Bestimmung des Peroxydasegehaltes der Leukocyten des peripheren Blutes als sehr geeignet. Bei zehn ausgewählten Patienten führte der Peroxydasegehalt pro myeloischer Zelle zu einer durchschnittlichen Extinktionsänderung von dE/dt $(t=15 \mathrm{Sek})=4,.23 \pm 1,14 \cdot 10^{-7}$; dieser Wert entsprach der Aktivität von $0,2777 \pm 0,152$ ng Meerrettichperoxydase.

\section{The photometric determination of myeloperoxidase with p-phenylendiamine}

The kinetics of myelo- and horseradish peroxidase have been investigated by means of a photometric-kinetic test using $p$-phenylendiamine as the H-Donor. Under certain conditions this assay seemed very suitable for the estimation of the peroxidase content of the peripheral blood leucocytes. A mean value of $\mathrm{dE} / \mathrm{dt}(t=15 \mathrm{sec})=4.23 \pm 1.14 \cdot 10^{-7}(=0.2777 \pm 0.152 \mathrm{ng}$ horseradish peroxidase $)$ per single myeloid cell was found in ten patients.

Bei hoher Substratspezifität $\left(\mathrm{H}_{2} \mathrm{O}_{2}\right.$, Methyl- und Äthylperoxid) besitzen die Peroxydasen ein weites Spektrum oxydabler H-Donatoren: Amine, Phenole, Guajakol, Leukofarbstoffe usf. Die Oxydationsgröße dieser Verbindungen ist für die einzelnen Peroxydasearten verschieden. $p$-Phenylendiamin kann von der Myeloperoxydase der Leukocyten sehr leicht oxydiert werden (1). $p$-Phenylendiamin wurde jedoch, soweit uns bekannt, für quantitativ biochemische Bestimmungen nur zum Nachweis peroxydatischer Aktivität in Milch und Gewebehomogenaten verwendet (2). $\mathrm{Da}$ es sich dabei um eine einfach und rasch durchführbare Methode handelt (2), sollte ihre Verwendbarkeit zur Bestimmung des Myeloperoxydasegehaltes der Leukocyten des peripheren Blutes untersucht werden. Dies schien uns besonders deshalb angezeigt, weil die Funktion dieses Fermentes trotz der sehr hohen Konzentration in den Granulocyten derzeit noch weitgehend unklar ist (3).

\section{Methodik}

Bereitung der Enzymproben: Zur Isolierung der Leukocyten wurden etwa $15 \mathrm{~m} /$ Heparinblut (bei sorgfältiger Präparation sind wenige $\mathrm{ml}$ ausreichend) zur Spontansedimentation bei Raumtemperatur aufgestellt. Nach ungefähr $1 \frac{1}{2} \mathrm{Stdn}$. wurde das überstehende Plasma abpipettiert. Restliche Erythrocyten wurden durch osmotischen Schock (30 Sek.) entfernt, die Leukocyten bei niedriger Tourenzahl $(110 \mathrm{~g})$ abzentrifugiert und in 3 bis $5 \mathrm{ml} 0,9$ proz. $\mathrm{NaCl}-L o ̈ s u n g$ suspendiert. Die Leukocytenzahl wurde durch Mehrfachzählung in der BürkER-Türkschen Zählkammer bestimmt und anschließend auf 5000-10000 Leukocyten $/ \mathrm{mm}^{3}$ eingestellt. Die Homogenisierung der Leukocytenisolate erfolgte mittels eines Ultra-Turrax (Typ 18/2; Janke \& Kunkel KG.). Es wurden jeweils $2 \mathrm{~m} /$ Suspension eingesetzt und $2 \mathrm{mal} 60$ Sek. im Eisbad homogenisiert.

Für die Berechnung des Myeloperoxydasegehaltes/myeloische Zelle wurden die Leukocytenisolate nach MAY-GRüNWALDGIEMSA gefärbt und jeweils 500 Zellen ausdifferenziert.
Photometrische Bestimmung ${ }^{1}$ :

Verwendete Reagenzien und Herstellung der Lösungen:

1. Wasserstoffperoxid, $30 \mathrm{proz}$. (w/v)

2. $p$-Phenylendiamin

3. Kaliumdihydrogenphosphat nach SöRENSEN

4. Di-Natriumhydrogenphosphat-2-Hydrat nach SöRENSEN.

a) Wasserstoffperoxid (6 mM)

Stammlösung ( $30 \mathrm{mM}$ ): $0,66 \mathrm{ml} 30$ proz. $\mathrm{H}_{2} \mathrm{O}_{2}$-Lösung mit bidest. Wasser auf $200 \mathrm{ml}$ verdünnen. Genaue Einștellung auf $0,03 \mathrm{M}$ $\left(10 \mathrm{ml} \quad 0,03 \mathrm{M} \mathrm{H}_{2} \mathrm{O}_{2} \rightleftharpoons 6 \mathrm{ml} \quad 0,1 \mathrm{~N} \mathrm{KMNO}_{4}\right)$. Stammlösung vor Gebrauch mit bidest. Wasser im Verhältnis $1: 5$ verdünnen (1 Teil Stammlösung +4 Teile bidest. Wasser).

b) $p$-Phenylendiaminlösung (1proz. w/v) Fa. British Drug Houses Ltd., Vertr. Schneider Chemie.

$100 \mathrm{mg} p$-Phenylendiamin in $10 \mathrm{ml}$ heißem bidest. Wasser lösen, filtrieren und rasch abkühlen.

c) Phosphatpuffer $1 / 15 \mathrm{M}, \mathrm{pH} 7,3$

$\mathrm{M} / 15 \mathrm{KH}_{2} \mathrm{PO}_{4}: 9,078 \mathrm{~g} / \mathrm{l}$

$M / 15 \mathrm{Na}_{2} \mathrm{PO}_{4}: 2 \mathrm{H}_{2} \mathrm{O}: 11,876 \mathrm{~g} / \mathrm{l}$

77,7 Vol.-\% M/15 Di-Natriumhydrogenphosphat im Gemisch.

\section{Bestimmungsansatz}

Meßstrahlung $492 \mathrm{~nm}$ (Photometer Eppendorf)

Meßtemperatur $25^{\circ}$ (thermokonstanter Küvettenhalter)

Meßvolumen $3 \mathrm{~m} /$, Schichtdicke $1 \mathrm{~cm}$.

Nacheinander in die Küvette pipettieren:

1. 2,5 (5) $\mathrm{m} /$ Phosphatpuffer $\mathrm{pH} 7,3$ (c)

2. $(0,05) 0,1 \mathrm{~m} /$ Leukocytenhomogenat

3. $0,2 \mathrm{ml} p$-Phenylendiaminlösung (b)

4. $0,2 \mathrm{~m} / \mathrm{H}_{2} \mathrm{O}_{2}$-Lösung (a);

rasch (etwa 3 Sek.) mischen und Extinktionszunahme alle 5 Sek. 15-30 Sek. lang messen. - Die Peroxydaseaktivität ergibt sich als $\mathrm{dE} / \mathrm{dt}$.

1) Bei der Herstellung der Lösungen wurde gegenüber den Angaben von $\mathrm{H}$. LücK (2) lediglich die $\mathrm{H}_{2} \mathrm{O}_{2}$-Konzentration geändert. 

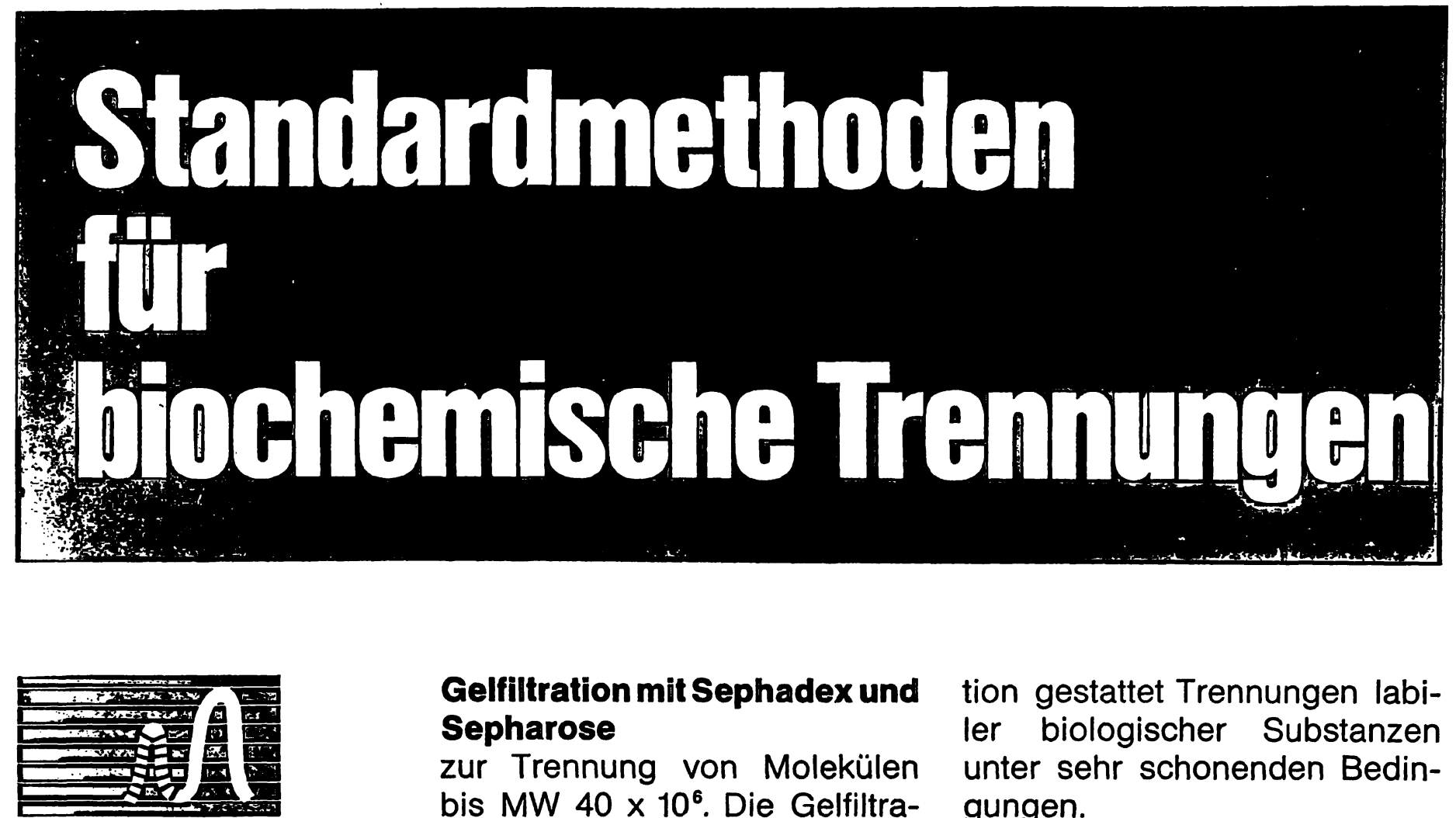

Gelfiltration mit Sephadex und Sepharose

zur Trennung von Molekülen bis MW $40 \times 10^{6}$. Die Gelfiltra-

tion gestattet Trennungen labiler biologischer Substanzen unter sehr schonenden Bedingungen.

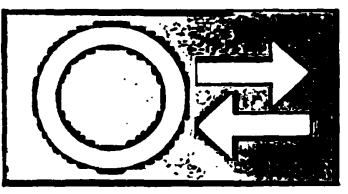

Ionenaustauscher-Chromato-

einigen. Charakteristisch sind graphie mit Sephadex-Ionenaustauschern,

die die Vorzüge von Celluloseund Kunstharzaustauschern ver-

hohe Kapazität, niedrige unspezifische Adsorption und ausgezeichnete Reproduzierbarkeit.

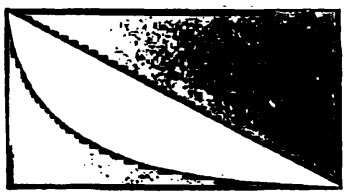

Verteilung in wäßrigen Phasen-Systemen mit Dextran und Dextran-Derivaten

zur Fraktionierung sehr hochmolekularer Stoffe wie Viren, Nukleinsäuren und Zellpartikeln unter sehr milden Bedingungen.

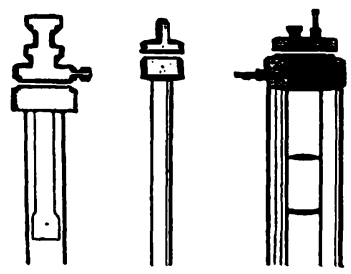

\section{Chromatographierohre.}

Unsere Chromatographierohre, die wir speziell für Gelfiltration und Ionenaustausch-Chromato- graphie. entwickelt haben, ermöglichen reproduzierbare Trennresultate. Sie stehen Ihnen in großer Auswahl mit diversem Zubehör zur Verfügung.

\section{Literaturdienst}

Als Hilfe für Wissenschaftler geben wir einen umfassenden Literaturdienst heraus. Eine jährlich erscheinende Referenzliste enthält etwa 1000 .neue
Literaturstellen. Bitte schreiben Sie uns, wenn wir Sie in unseren Verteiler aufnehmen sollen. Fordern Sie auch Broschüren über unsere Separationsprodukte und das Literaturverzeichnis an. 


\section{ANIIOSAUREN-ANALYSATOR}

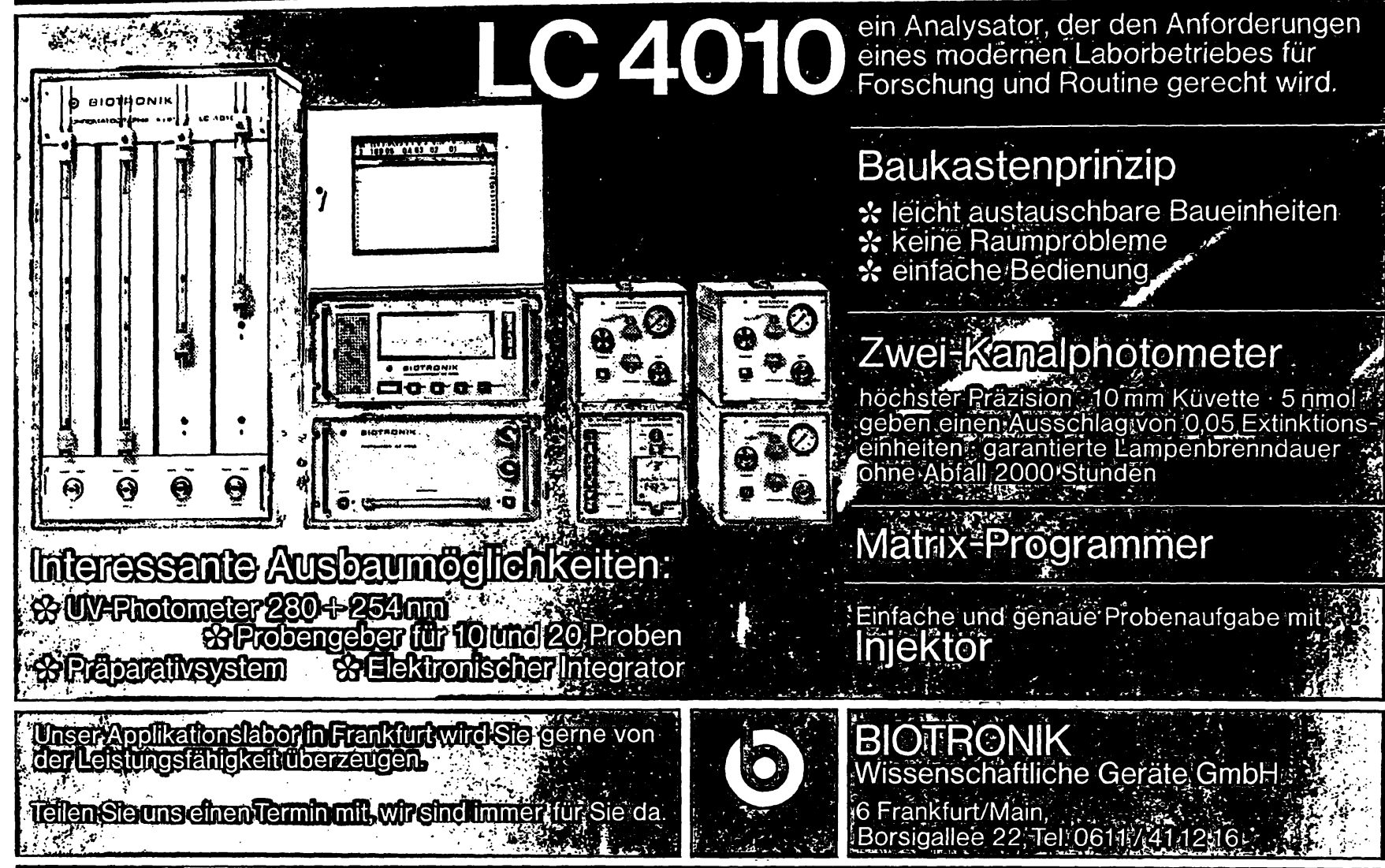

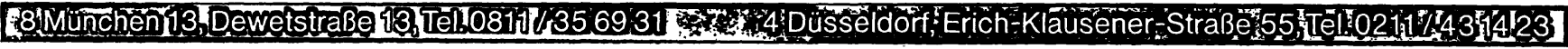

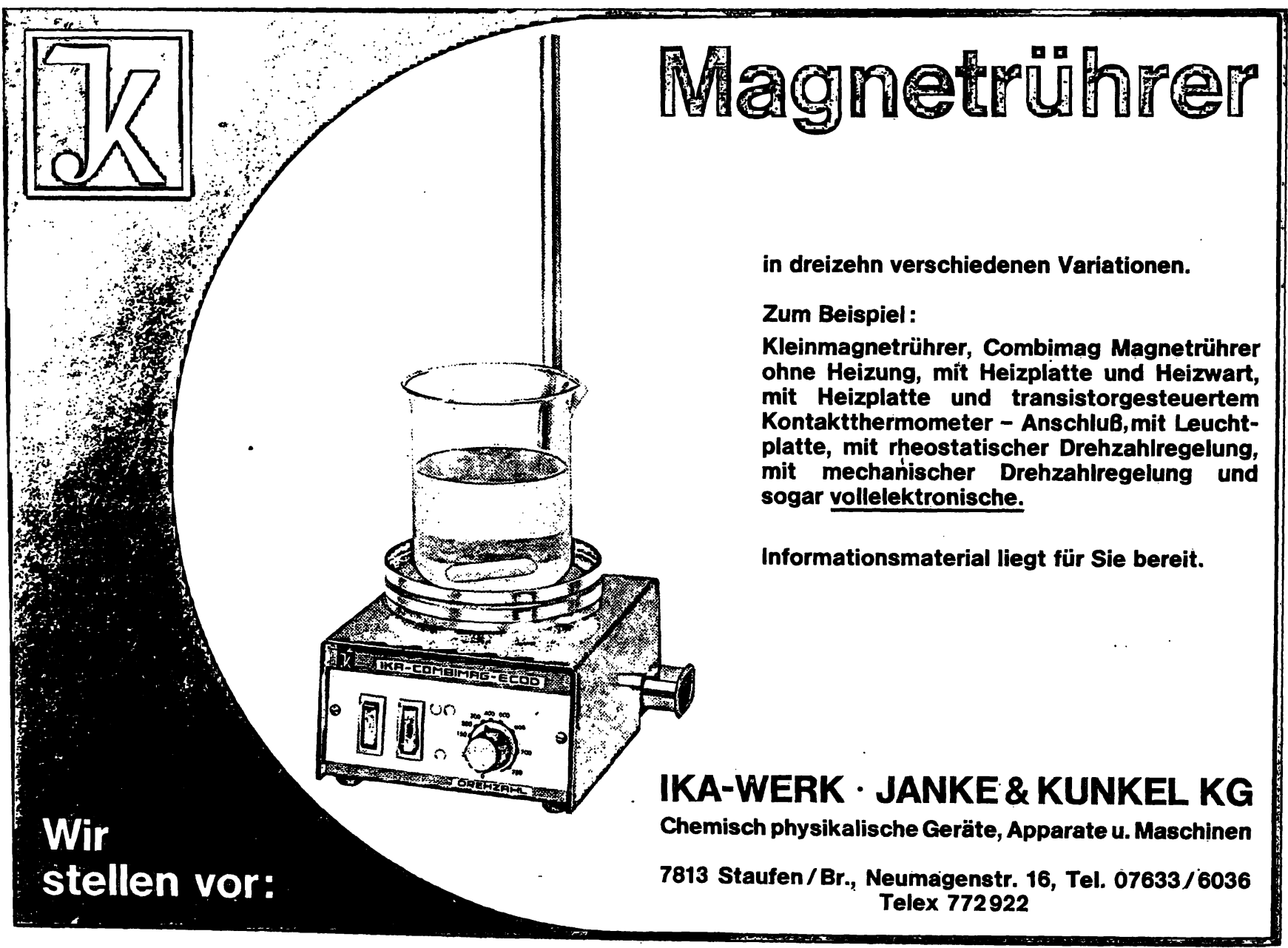


Kontrollansätze: Die Extinktionsänderung ohne Zusatz von $\mathrm{H}_{2} \mathrm{O}_{2}$ betrug im Durchschnitt $1,7 \%$ des $\mathrm{H}_{2} \mathrm{O}_{2}$-haltigen Ansatzes. Ohne Zusatz von $p$-Phenylendiamin ergab sich keine Extinktionsänderung.

Meerrettichperoxydase

Verwendet wurde gereinigte Meerrettichperoxydase der Fa. Bochringer \& Söhne GmbH. Mannheim (Nr. 15301 EPAA). Am oben angeführten Bestimmungsansatz der Myeloperoxydase wurde zur Bestimmung der Meerrettichperoxydase-Standardkurve lediglich der pH-Bereich des Phosphatpuffers auf $\mathrm{pH} \mathrm{5,2}$ geändert.

\section{Ergebnisse}

Einfluß der Homogenisierdauer auf die Myeloperoxydase-Aktivität der Leukocytenhomogenate

Wie aus Tabelle 1 ersichtlich, wurde die höchste Aktivität nach einer Homogenisierdauer von $2 \mathrm{mal} 60 \mathrm{Sek}$. erreicht. Das eingesetzte Volumen der Leukocytensuspension betrug $2 \mathrm{ml}$, die Zellzahl lag zwischen 4400 und 12000 Leukocyten $/ \mathrm{mm}^{3}$. Längere Homogenisierdauer führte in einzelnen Fällen zu geringem Aktivitätsverlust. Bei einer Zellzahl unter $5000 / \mathrm{mm}^{3}$ wurde das Aktivitätsmaximum schon nach einer Homogenisierdauer von 60 Sek. erreicht.

Tab. 1

Einfluß der Homogenisierdauer auf die Myeloperoxydase-Aktivität

\begin{tabular}{|c|c|c|c|c|c|c|c|}
\hline \multirow{2}{*}{ 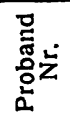 } & \multicolumn{6}{|c|}{$\begin{array}{l}\text { Extinktionsänderung dE/dt nach Homogenisier- } \\
\text { dauer in Sek. }\end{array}$} & \multirow{2}{*}{$\begin{array}{l}\text { Zellzahl } \\
\text { pro } \mathrm{mm}\end{array}$} \\
\hline & 20 & 40 & 60 & 120 & 180 & 240 & \\
\hline $\begin{array}{l}1 \\
2 \\
3 \\
4 \\
5 \\
6 \\
7\end{array}$ & $\begin{array}{l}0,15 \\
0,20 \\
0,17 \\
0,27 \\
0,155 \\
0,29 \\
0,19\end{array}$ & $\begin{array}{l}0,30 \\
0,25 \\
0,31 \\
0,37 \\
0,35 \\
0,37 \\
0,34\end{array}$ & $\begin{array}{l}0,365 \\
0,34 \\
0,44 \\
0,39 \\
0,44 \\
0,39 \\
0,39\end{array}$ & $\begin{array}{l}0,37 \\
0,35 \\
0,50 \\
0,39 \\
0,47 \\
0,435 \\
0,42\end{array}$ & $\begin{array}{l}0,37 \\
0,35 \\
0,50 \\
0,385 \\
0,435 \\
0,42 \\
0,40\end{array}$ & $\begin{array}{l}0,37 \\
0,34 \\
0,47 \\
0,39 \\
0,43 \\
0,42 \\
0,40\end{array}$ & $\begin{array}{r}8100 \\
9000 \\
7500 \\
4400 \\
8000 \\
6500 \\
12000\end{array}$ \\
\hline
\end{tabular}

\section{Kinetische Untersuchungen}

Es wurde eine Reihe von Versuchsbedingungen, welche die $p$-Phenylenumsetzung beeinflussen, sowohl für die Myeloperoxydase als auch für die Meerrettichperoxydase geprüft.

Myeloperoxydase

Zeitumsatqkurve und Abbängigkeit der Aktivität vom $p H$ Der Verlauf der Zeitumsatzkurve ist vom $\mathrm{pH}$ abhängig (Abb. 1). Bei $\mathrm{pH} 7,3$ nimmt die Extinktionsänderung $\mathrm{dE} / 5$ Sek. schon während der ersten 15 Sek., wenn auch nur gering, so doch stetig ab. Lediglich bei $\mathrm{pH} \mathrm{6,6}$ verläuft die Reaktion während der ersten 10 Sek. linear. Bei $\mathrm{pH} 7,8$ und $\mathrm{pH} 8,0$ ist die Extinktionsänderung der ersten 5 Sek. kleiner als die nächst folgenden Meßwerte.

Die Extinktionsänderung nach 15 Sek. ist bei pH 8,0 eben so groß wie bei pH 5,4. Nach einer Versuchsdauer von 30 Sek. ist jedoch der im Alkalischen gemessene Wert bereits wesentlich höher; dieser Unterschied wird bis zum Versuchsende immer größer (Abb. 2a).

Dementsprechend ist der Verlauf der pH-Aktivitätskurven je nach Reaktionsdauer verschieden (Äbb. 2a und $2 \mathrm{~b}$ ). Nach 5 Sek. sind 2 Aktivitätsgipfel, und zwar bei

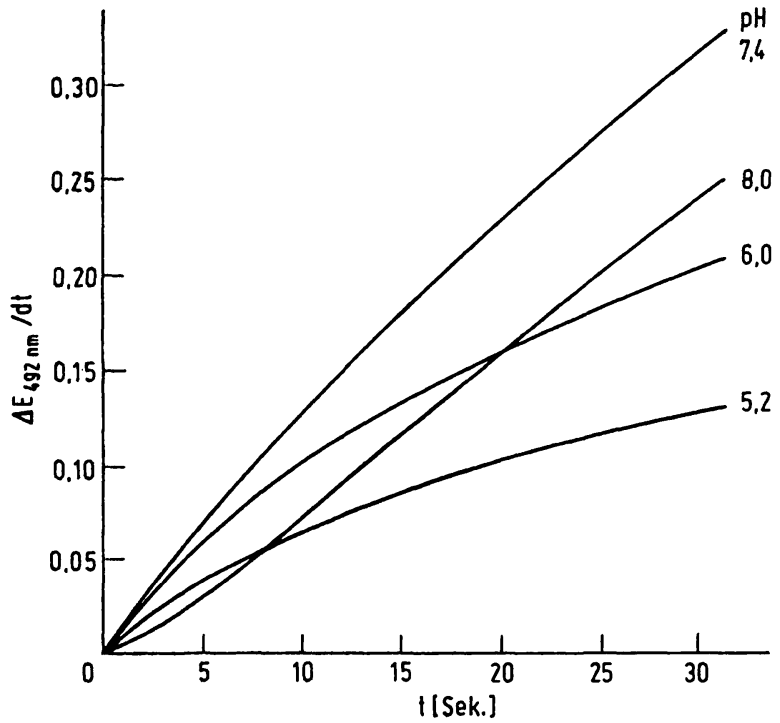

Abb. 1

Zeitumsatzkurven bei verschiedenem pH-Versuchsbedingungen: $M / 15$ Phosphatpuffer; $p$-Phenylendiamin-Endkonzentration $6,13 \mathrm{~mm}$; $\mathrm{H}_{2} \mathrm{O}_{2}$-Endkonzentration $0,4 \mathrm{~mm} ; 100 \mu l$ Leukocytenhomogenat (5000 Leukocyten $/ \mathrm{mm}^{2}$ ).

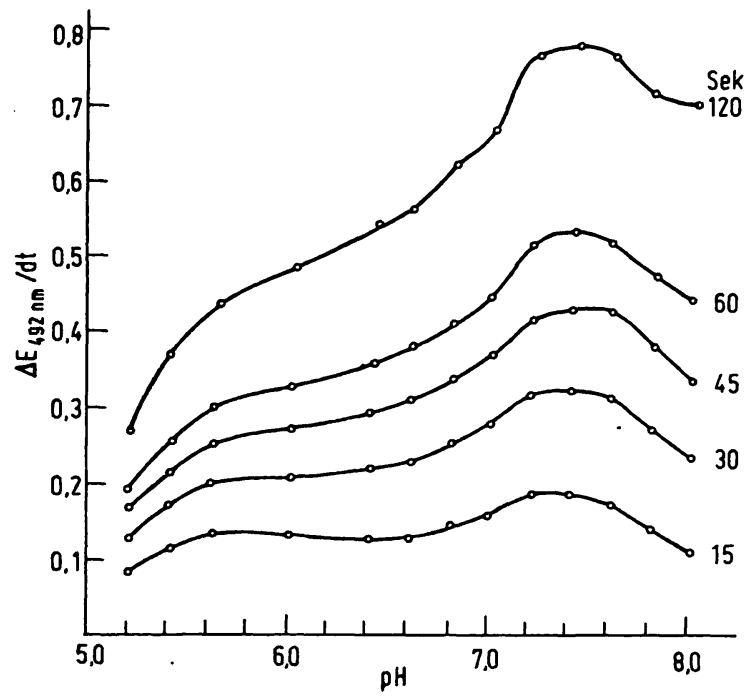

Abb. $2 a$

Verlauf der pH-Aktivitätskurven in Abhängigkeit vom Zeitpunkt der Messung. Versuchsbedingungen: $M / 15$ Phosphatpuffer; $p$-Phenylendiamin-Endkonzentration $6,13 \mathrm{mM} ; \mathrm{H}_{2} \mathrm{O}_{2}$-Endkonzentration $0,4 \mathrm{~mm}$ Enzymmenge $100 \mu l$ Leukocytenhomogenat (Zellzahl $5000 / \mathrm{mm}^{3}$ )

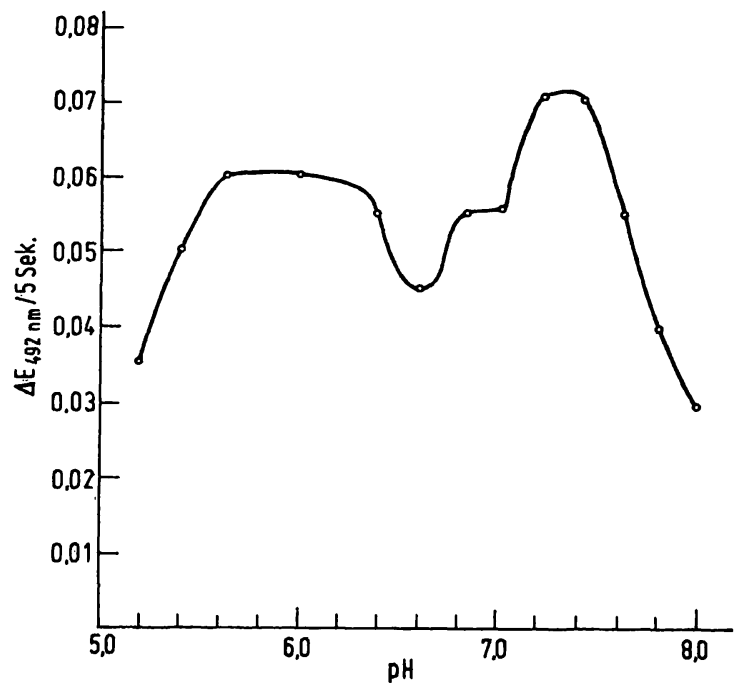

Abb. 2b

Verlauf der pH-Aktivitätskurve nach einer Meßdauer von 5 Sek. Versuchsbedingungen: siehe $A b b, 2 a$ 
pH 5,6-6,0 und bei pH 7,2-7,4 zu erkennen (Abb. 2b). Bei weiterer Beobachtung zeigt sich nur mehr das Aktivitätsoptimum im alkalischen Bereich. Der Aktivitätsverlust bei pH-Werten über 7,4 wird jedoch mit zunehmender Versuchsdauer gegenüber der Aktivitätszunahme von $\mathrm{pH} 5,2-7,3$ immer geringer.

\section{Abbängigkeit der Reaktion von der $\mathrm{H}_{2} \mathrm{O}_{2}$-Konzentration}

Die Zeitumsatzkurven sind auch, wie aus Abbildung 3 ersichtlich, je nach $\mathrm{H}_{2} \mathrm{O}_{2}$-Konzentration verschieden. Dies ist zum Teil auf eine durch überschüssiges $\mathrm{H}_{2} \mathrm{O}_{2}$ verursachte Hemmung der Myeloperoxydase zurückzuführen. Das Ausmaß des Hemmeffektes ist von der Versuchsdauer abhängig (Abb. 4). Bei einer Endkonzentration von $0,1 \mathrm{mM}$ (Zusatz von $0,05 \mathrm{~m} l$ der $6 \mathrm{mM} \mathrm{H}_{2} \mathrm{O}_{2^{-}}$ Lösung) wird der maximale Extinktionswert schon nach 90 Sek. erreicht, da die $\mathrm{H}_{2} \mathrm{O}_{2}$-Konzentration den Umsatz limitiert. Die optimale Substratkonzentration ist von der Meßdauer unabhängig und liegt unter den angegebenen Bedingungen bei $0,4 \mathrm{~mm}$ (Zusatz von $0,2 \mathrm{ml}$ der $6 \mathrm{mM} \mathrm{H}_{2} \mathrm{O}_{2}$-Lösung) (Abb. 3 und Abb. 4).

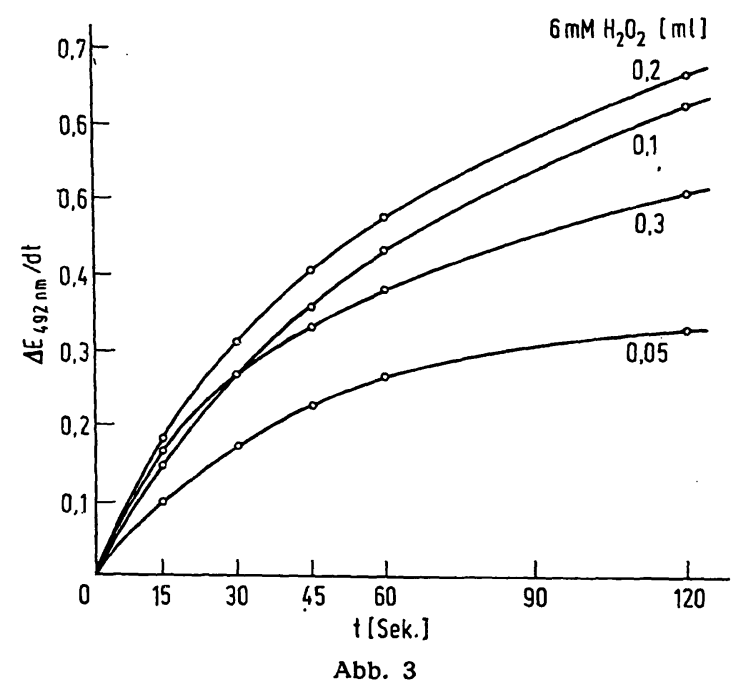

Abhängigkeit der Myeloperoxydaseaktivität von der $\mathrm{H}_{2} \mathrm{O}_{2}$-Konzentration: Zeitumsatzkurven. Bedingungen: $\mathrm{M} / 15$ Phosphatpuffer;

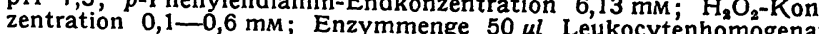
(Zellzahl $11500 / \mathrm{mm}^{3}$ )

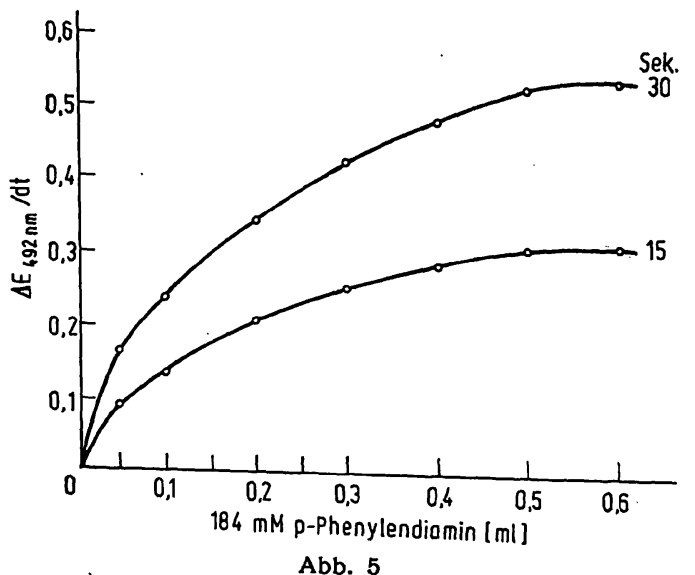

Abhängigkeit der Reaktionsgröße dE/dt $(t=15$ Sek. und 30 Sek.) von der $p$-Phenylendiaminkonzentration. Bedingungen: $M / 15$ Phosphatpuffer $\mathrm{pH} 7,3 ; \mathrm{H}_{2} \mathrm{O}_{2}$-Endkonzentration $0,4 \mathrm{mM} ; p$-Phenylendiacytenhomogenat (Leukocytenzahi $4000 / \mathrm{mm}^{3}$ )
Abbängigkeit der Reaktion von der p-Pbenylendiaminkonzen- . tration

Unter den gewählten Bedingungen wird die $p$-Phenylendiamin-Sättigungsdosis bei Zusatz von $0,5 \mathrm{~m} l$ einer 2 proz. $p$-Phenylendiaminlösung ( $30,7 \mathrm{~mm}$ Endkonzentration) erreicht, und zwar sowohl für eine Versuchsdauer von 15 Sek. als auch von 30 Sek. (Abb.5). Die eingesetzte Enzymmenge führte dabei zu einer Extinktionsänderung von $\mathrm{dE} / 15$ Sek. $=0,33$. Unter Standardbedingungen $(0,2 \mathrm{~m} l$ einer 1.proz. p-Phenylendiaminlösung) führt dieselbe Enzymkonzentration zu einer Extinktionsänderung von 0,15 . Als Michaeliskonstante $K_{m}$ ergibt sich eine $p$ Phenylendiamin-Endkonzentration von 7,36 mM.

\section{Abbängigkeit der Reaktion von der Enzymkonzentration}

Bei Einsatz von 25-200 $\mu$ l Probe aus Leukocytensuspensionen mit einer Zellzahl zwischen 5500 und 6500 Zellen $/ \mathrm{mm}^{3}$ war die Extinktionszunahme der Enzymkonzentration unter den gewählten Bedingungen während der ersten 15 Sek. direkt proportional. Bei einer Versuchsdauer von 30 Sek. kam es ab dE/30 Sek. größer als 0,45 zur Abweichung von der Linearen (Abb. 6).

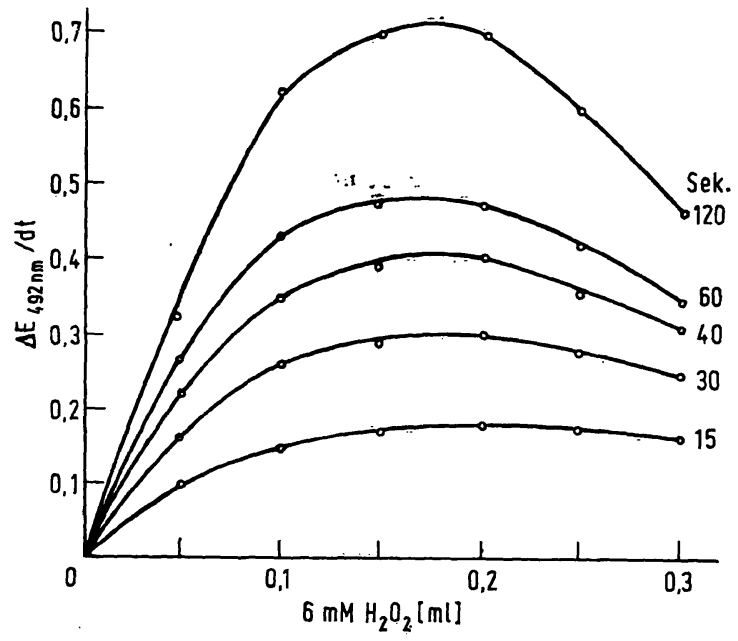

Abb. 4

Abhängigkeit der Myeloperoxydaseaktivität von der $\mathrm{H}_{2} \mathrm{O}$-Konzentration. Beobachtungsdauer $15-120 \mathrm{Sek}$. Bedingungen siehe Abb. 3

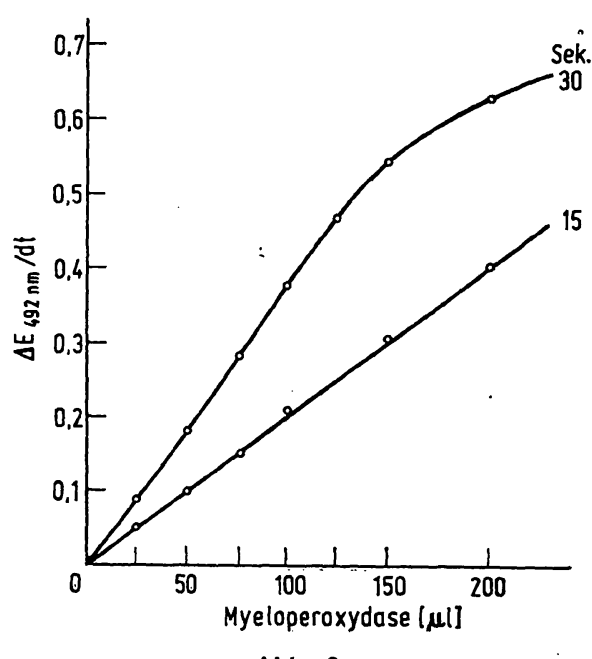

Abhängigkeit der Extinktionsänderung $\mathrm{dE} / \mathrm{dt}$ von der Myeloperoxydasekonzentration. Versuchsbedingungen: $M / 15$ Phosphatpuffer $\mathrm{pH}$ 7,3; p-Phenylendiamin-Endkonzentration $6,13 \mathrm{~mm} ; \mathrm{H}_{2} \mathrm{O}_{2}$-Endkonzentration $0,4 \mathrm{~mm}$; Enzymkonzentration: $25-200 \mu l$ Leukocyten- 


\section{Meerrettichperoxydase}

Zur Bestimmung einer Standardkurve wurden entsprechende Untersuchungen auch für Meerrettichperoxydase durchgeführt. Das pH-Optimum lag für Meerrettichperoxydase bei pH 5,2-5,4 (Abb. 7). Die Aktivitätsabnahme bei steigendem $\mathrm{pH}$ wurde mit zunehmender Versuchsdauer immer größer. Der Bereich des $\mathrm{pH}-$ Optimum blieb bis $\mathrm{zu}$ einer Versuchsdauer von 120 Sek. unverändert.

Bei Einsatz von $0,2 \mu \mathrm{g}$ Meerrettichperoxydase wurde die $p$-Phenylendiamin-Sättigungsdosis unter den angeführten Bedingungen bei einer Konzentration von $3,07 \mathrm{~mm}$ erreicht $(0,1 \mathrm{~m} l$ einer 1 proz. $p$-Phenylendiamin Lösung) (Abb. 6).

Bei steigender $\mathrm{H}_{2} \mathrm{O}_{2}$-Konzentration (Endkonzentration $0,1 \mathrm{~mm}$ bis $1,0 \mathrm{~mm}$ ) war bei Einsatz von $0,2 \mu \mathrm{g}$ Meerrettichperoxydase keine Hemmung der Aktivität zu erkennen. Bei Einsatz von $0,1 \mu \mathrm{g}$ wurden nach Zusatz von $0,3 \mathrm{ml}, 0,4 \mathrm{~m} l$ und $0,5 \mathrm{~m} l \mathrm{H}_{2} \mathrm{O}_{2}$-Lösung ( $6 \mathrm{~mm}$ ) die gleichen Extinktionsänderungen gemessen (Abb. 9a, 9b und 9c).

Standardkurve: Zur Bestimmung der $\mathrm{dE} / \mathrm{dt}$ Abhängigkeit von der eingesetzten Meerrettichperoxydasemenge wurde im Bestimmungsansatz der Myeloperoxydase (siehe Methodik) nur der $\mathrm{pH}$-Bereich entsprechend dem $\mathrm{pH}$ Optimum der Meerrettichperoxydase geändert. Die Aktivitätskurven für eine Versuchsdauer von 15 Sek. und 30 Sek. sind in Abbildung 10 dargestellt. Es sind dies die Bezugskurven für die Angaben der Myeloperoxydaseaktivität in ng Meerrettichperoxydase.

\section{Peroxydasegebalt myeloischer Zellen des peripheren Blutes}

Unter den eingangs angeführten Versuchsbedingungen wurde der Myeloperoxydasegehalt/myeloischer Zelle bei zehn Patienten im Alter von 18-76 Jahren, deren Blut-

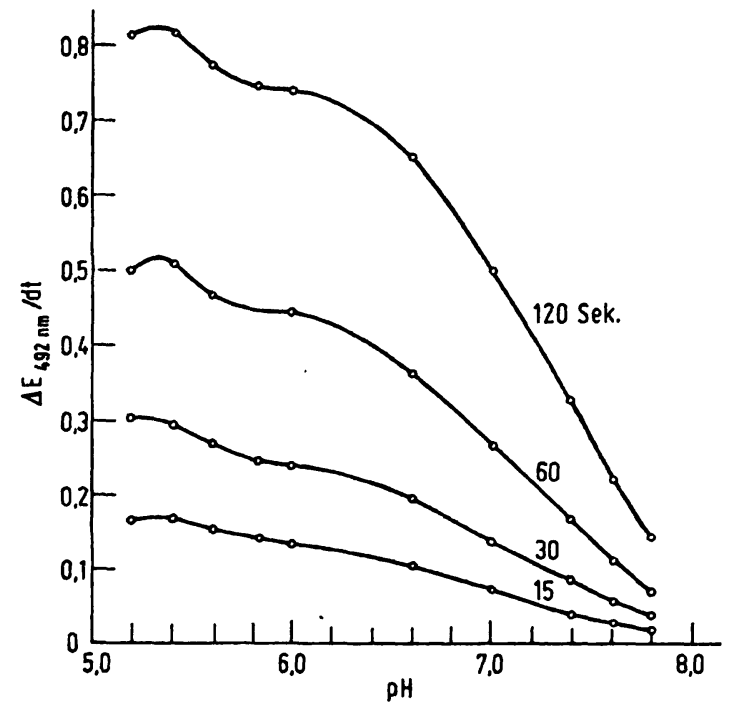

Abb. 7

Verlauf der pH-Aktivitätskurve in Abhängigkeit vorn Zeitpunkt der Messung. Bedingungen: $M / 15$ Phosphatpuffer; $\mathrm{pH} 7,3 ; p$-Phenylendiamin-Endkonzentration $6,13 \mathrm{mM} ; \mathrm{H}_{2} \mathrm{O}_{2}$-Endkonzentration $0,4 \mathrm{mM}$; Enzymmenge 0,1 $\mathrm{\mu g}$ Meerrettichperoxydase

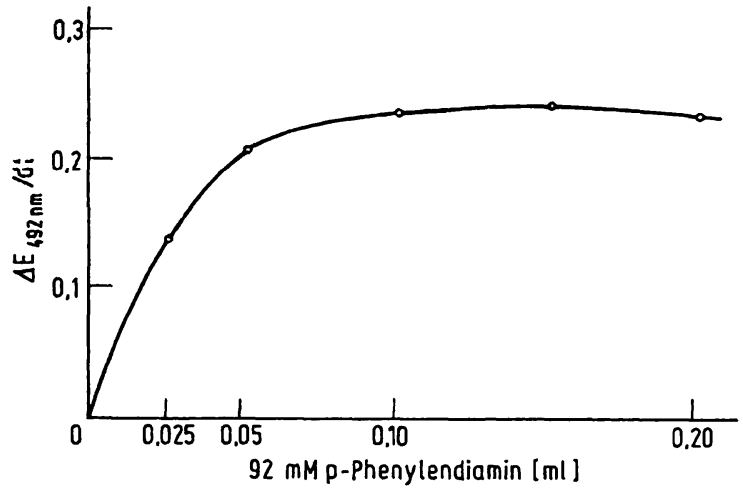

Abb. 8

Abhängigkeit der Reaktionsgröße dE/dt $(t=15$ Sek.) von der $p$ Phenylendiaminkonzentration. Bedingungen: $M / 15$ Phosphatpuffer pH 5,2; $\mathrm{H}_{2} \mathrm{O}_{2}$-Endkonzentration $0,4 \mathrm{mM}$;Enzymmenge $0,2 \mu \mathrm{g}$ Meer rettichperoxydase; $p$-Phenylendiamin-Endkonzentration von 0,765 bis $6,13 \mathrm{mM}$

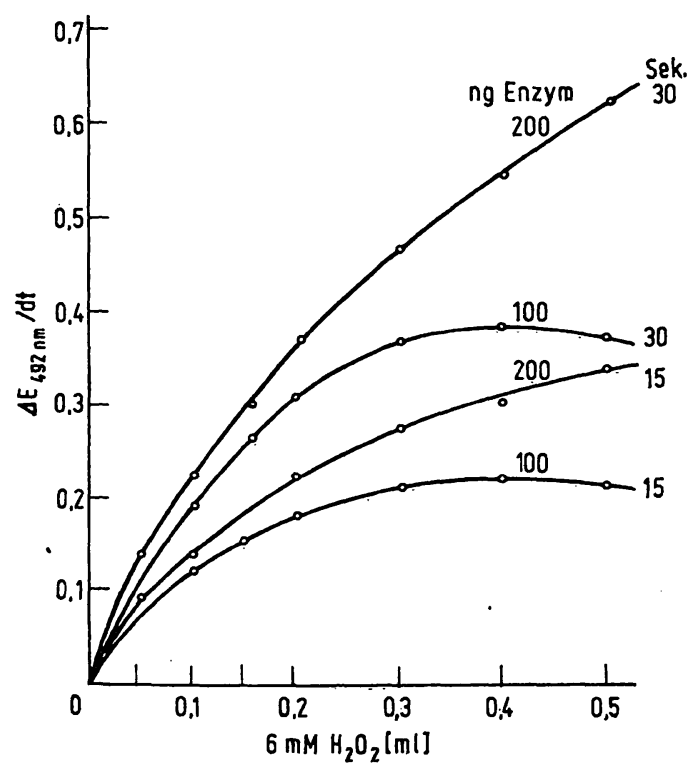

Abb. 9 a

Abhängigkeit der Aktivität der Meerrettichperoxydase von der $\mathrm{H}, \mathrm{O}_{2}$-Konzentration. Meßdauer 15 Sek. und 30 Sek.; Bedingungen: $M / 15$ Phosphatpuffer $\mathrm{pH}$. 5,2 ; Endkonzentration -Endkonzentration $6,13 \mathrm{~mm} ; \mathrm{H}_{2} \mathrm{O}_{2}$ tration 0,1 und $0,2 \mu \mathrm{g}$ Meerrettichperoxydase/Ansatz

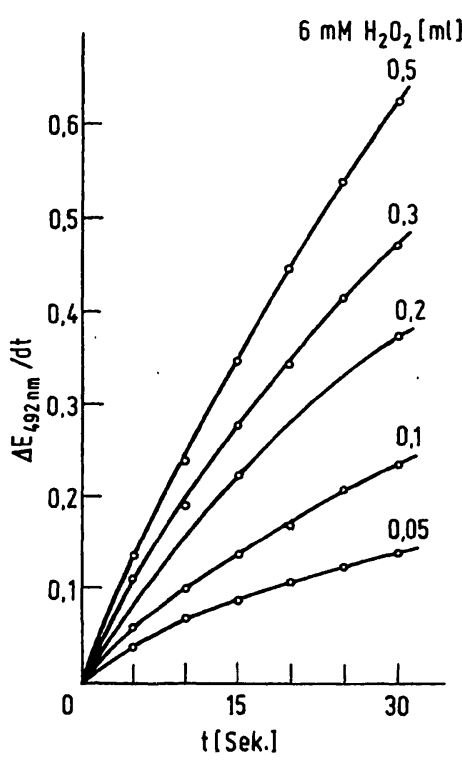

Abb. 9b

Zeitumsatzkurven bei verschiedener $\mathrm{H}_{2} \mathrm{O}_{2}$ Konzentration. Bedingungen: $M / 15$ Phosphatpuffer $\mathrm{pH} 5,2 ; p$-Phenylendiamin-Endkonzentration 6,13 mM; Enzymkonzentration $0,2 \mu \mathrm{g}$ Meerrettichperoxydase; $\mathrm{H}_{3} \mathrm{O}_{4}-$

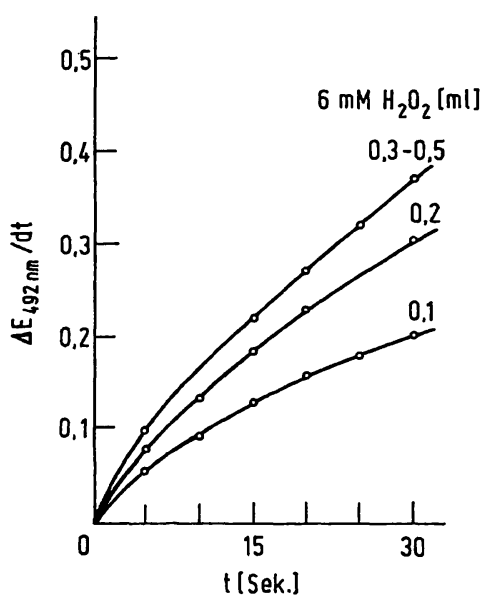

Abb. 9c

Zeitumsatzkurven bei verschiedener $\mathrm{H}_{2} \mathrm{O}_{2}$-Konzentration. Enzymkonzentration $0,1 \mu \mathrm{g}$ Meerrettichperoxydase;
i. ü. gegenüber Legende zu Abb. $9 \mathrm{~b}$ unverändert 
Tab. 2

Peroxydasegehalt myeloischer Zellen des peripheren Blutes

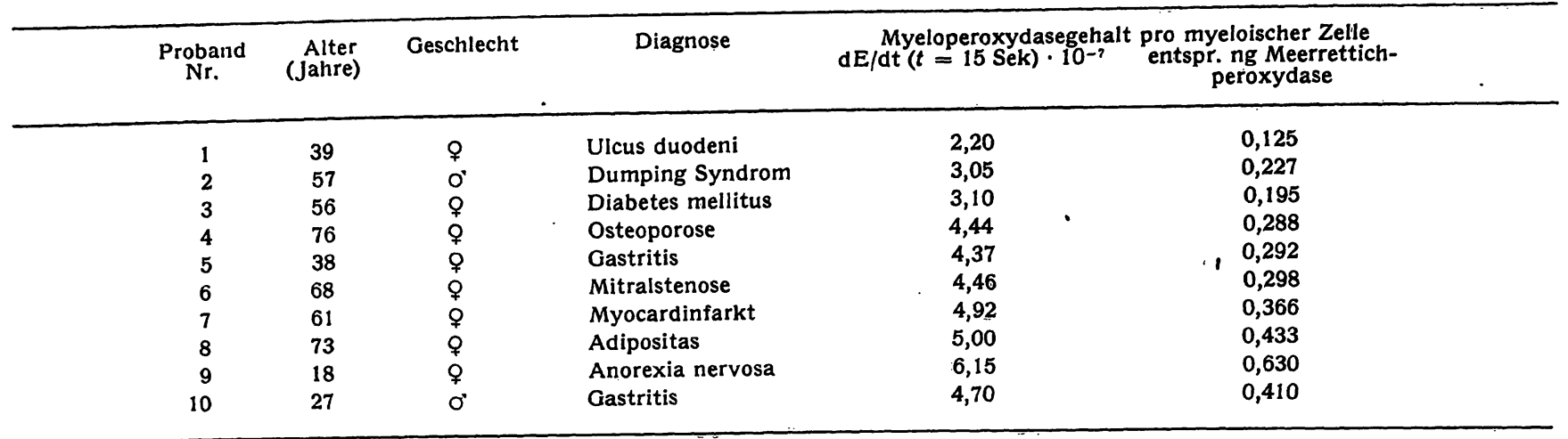

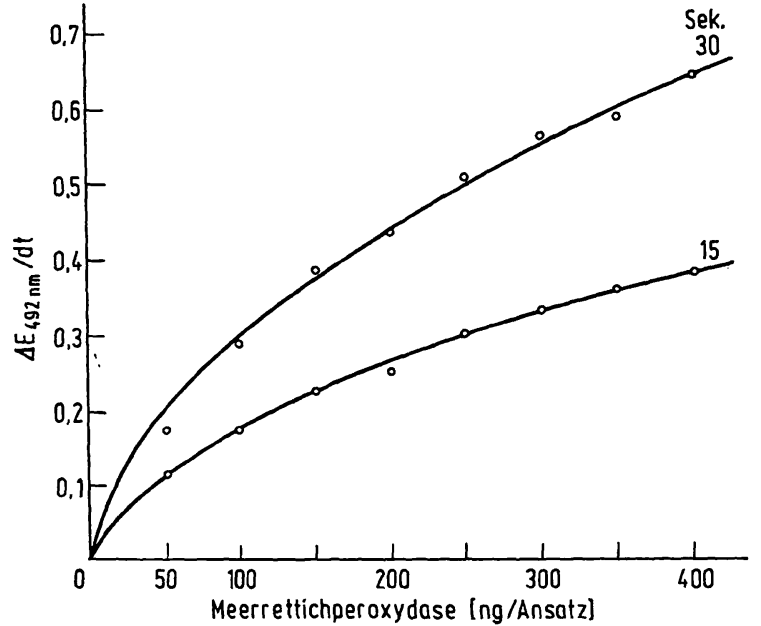

Abb. 10

Abhängigkeit der Extinktionsänderung $\mathrm{dE} / \mathrm{dt}(t=15 \mathrm{Sek}$. und 30 Sek.) von der Meerrettichperoxydase-Konzentration. Bedingungen: M/15 Phosphatpuffer pH 5,$2 ;$ p-Phenylendiamin-Endkonzentration $6,13 \mathrm{mM} ; \mathrm{H}_{2} \mathrm{O}_{2}$-Endkonzentration $0,4 \mathrm{mM}$; MeerrettichperoxydaseKonzentration von $0,05-0,4 \mu \mathrm{g}$ Meerrettichperoxydase

bild der Norm entsprach, bestimmt (Tab. 2). Es fand sich ein durchschnittlicher Wert von $\mathrm{dE} / 15$ Sek. $=4,23 \pm 1,14 \cdot 10^{-7}$; dies entsprach der Aktivität von $0,277 \pm 0,152 \mathrm{ng}$ Meerrettichperoxydase.

\section{Diskussion}

Zur quantitativen Bestimmung der Peroxydaseaktivität kann der Substratverbrauch oder die Oxydation eines H-Donators herangezogen werden: Guajakol $(3,12,9)$, Leukofarbstoffe $(10,16), \alpha$-Naphthol-Benzidin (4), Dimethyl-p-Phenylendiamin $(14,6)$, Pyrogallol (15) usf. $p$-Phenylendiamin wurde schon von K. AGNER, dex als erster die Peroxydase der Leukocyten isolierte, als leicht oxydabler H-Donator der Myeloperoxydase beschrieben (1). Es wird dabei in eine farbige Molekülverbindung aus Diamin und Diimin umgewandelt (2).

$p$-Phenylendiamin wurde bis jetzt, soweit uns bekannt, für quantitative Messungen nur zur• Bestimmung der Peroxydase in Milch und Gewebehomogenaten verwendet $(2,7,13,14)$. Unter bestimmten Versuchsbedingungen erwies sich nun diese photometrische Bestimmungsmethode auch zur quantitativen Er- fassung der Myeloperoxydase sehr geeignet: Innerhalb bestimmter Grenzen herrscht strenge Linearität zwischen Enzymkonzentration und Extinktionsänderung, die Durchführung der Messung erfolgt rasch und einfach.

Die Enzymkonzentration ergibt sich als dE/dt. Da gereinigte Myeloperoxydase kaum erhältlich ist, wurde zur Gewinnung einer Standardkurve Meerrettichperoxydase verwendet (siehe 1. c. (9)). Myeloperoxydase und Meerrettichperoxydase sind jedoch nicht nur in ihren physikalischen Eigenschaften, sondern auch in ihrer Kinetik verschieden. In unseren Versuchen zeigte sich dies bezüglich der Lage des pH-Optimum, der in keinem pH-Bereich nachweisbaren initialen Hemmung der Merrettichperoxydase, der bei steigender $\mathrm{H}_{2} \mathrm{O}_{2}$-Konzentration später einsetzenden Hemmung der Meerrettichperoxydase und der viel höheren $p$-Phenylendiamin-Sättigungsdosis für die Myeloperoxydase. Im Gegensatz zur Meerrettichperoxydase besteht zwischen der Extinktionsänderung $\mathrm{dE}$ und der Myeloperoxydasekonzentration innerhalb bestimmter Grenzen strenge Linearität.

Die Angabe der Myeloperoxydaseaktivität in ng Meerrettichperoxydase ist somit u. a. von den - willkürlich festgelegten - Versuchsbedingungen zur Erstellung der Meerrettichperoxydase-Standardkurve abhängig. Für die Bestimmung des Myeloperoxydasegehaltes pro myeloischer Zelle - Lymphocyten sind peroxydasenegativ (15) - wurden Patienten ausgewählt, deren peripheres Blutbild der Norm entsprach, und die nicht an einem Malignom erkrankt waren (5). Es wurde ein Durchschnittswert entsprechend 0,277 $\pm 0,152$ ng Meerrettichperoxydase/myeloischer Zelle errechnet. Die Abweichung der Einzelergebnisse vom Mittelwert ist, wenn man von den Fällen 1 und 9 (18 Jahre - s. (5)) bei unserem ausgewählten Patientengut nicht größer als bei den Normalfällen von N. Katsushima (9); die gefundenen Werte dürften Normalwerten entsprechen. Ein Vergleich mit den Angaben anderer Autoren $(9,3)$ ist auf Grund der unterschiedlichen Methodik leider unmöglich. Bei Verwendung des Guajakoltestes wurden nach einer Reaktionszeit von 60 Sek. zwei pH-Optima der Myeloperoxydase nachgewiesen (17). Das Vorliegen eines $\mathrm{pH}-O$-Optimum im sauren Bereich führte zu verschiedenen Vermutungen über die intrazelluläre Funk- 
tion der Myeloperoxydase. Bei unseren Versuchen war ein solches $\mathrm{pH}-$ Optimum im Sauren nur am Beginn des Reaktionsablaufes festzustellen; es scheint demnach eher methodisch bedingt zu sein.
Unter Verwendung der beschriebenen Methode werden zur Zeit umfangreiche Untersuchungen des Peroxydasegehaltes der Leukocyten verschiedener klinischer Krankheitsbilder durchgeführt.

\section{Literatur}

1. Agner, K., Acta physiol. Scand. 8, 1 (1941). - 2. Lück, H., in: Methoden der enzymatischen Analyse, hrsg. von H.-V. Bergmeyer Verlag Chemie, Weinheim (1962). - 3. Schustz, J. und K. Kaminker, Arch. Biochem. Biophysics 96, 465 (1962). 4. Chance, B. und A. C. Maehly, in: Colowick, S. P. und N. O. Kaplan, Vol. II., Method in Enzymology Academie Press, New York (1955). - 5. Schultz, J., A. Gordon und N. SfiAy, J. Amer. chem. Soc. 79, 1932 (1957). - 6. LoEB, H. G. und R. Doniger, Brit. J. Cancer 12, 669 (1958). - 7. Neufeld, H. A., F. V. Lucas, A. P. Marbin und E. Stotz, Cancer Res. 15, 550 (1955). - 8. WrLlstätrer, R. und A. Weber, Liebigs Ann. Chem. 449, 156 (1926). - 9. Karsushrma, N., Tohoku J. Exper. Med.
78, 339 (1963). - 10. Straus, W., J. Biophys. Biochem. Cytol. 4, 541 (1958). - 11. Kelenyt, G., J. Pongratz, S. Orban und G. Deak, Blood 18, 417 (1961). - 12. WillstätTER, R. und A. Stoll, Liebigs Ann. Chem. 21, 416 (1918). - 13. Aurand, L. W., M. W. Roberts und J. T. Cardwell, J. Dairy Sci. 39, 568 (1956). - 14. Kiermeier, F. und C. Kayser, Z. Lebensmittelunters. und -Forsch. 112, 481 (1960). - 15. WINTROBE, M. M., Clinical Hcmatology, $5^{\text {th }}$ edition, Lea \& Febiger, Philadelphia, Pennsylvania (1961). - 16. Katsushima, N., Tohoku J. Exper. Med. 79, 250 (1963). - 17. Rohrer, G. F., J. P. WARTBURG und H. AEBr, Biochem. Z. 344, 492 (1966).

Doz. Dr. H. Pietschmann

A 1095 Wien

Garnisongasse 13 